
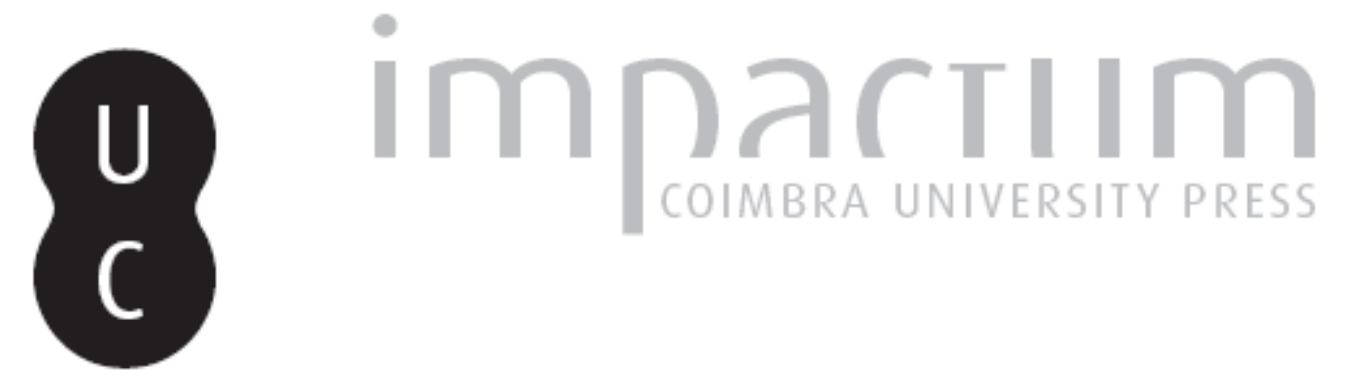

\title{
«Los nombres 'itálicos' de los Astures meridionales»
}

\section{Autor(es): $\quad$ Prósper, Blanca María}

Publicado por: Faculdade de Letras da Universidade de Coimbra

URL persistente:

URI:http://hdl.handle.net/10316.2/37769

DOI:

DOI:http://dx.doi.org/10.14195/1647-8657_47_8

Accessed : $\quad$ 26-Apr-2023 11:49:53

A navegação consulta e descarregamento dos títulos inseridos nas Bibliotecas Digitais UC Digitalis, UC Pombalina e UC Impactum, pressupõem a aceitação plena e sem reservas dos Termos e Condições de Uso destas Bibliotecas Digitais, disponíveis em https://digitalis.uc.pt/pt-pt/termos.

Conforme exposto nos referidos Termos e Condições de Uso, o descarregamento de títulos de acesso restrito requer uma licença válida de autorização devendo o utilizador aceder ao(s) documento(s) a partir de um endereço de IP da instituição detentora da supramencionada licença.

Ao utilizador é apenas permitido o descarregamento para uso pessoal, pelo que o emprego do(s) título(s) descarregado(s) para outro fim, designadamente comercial, carece de autorização do respetivo autor ou editor da obra.

Na medida em que todas as obras da UC Digitalis se encontram protegidas pelo Código do Direito de Autor e Direitos Conexos e demais legislação aplicável, toda a cópia, parcial ou total, deste documento, nos casos em que é legalmente admitida, deverá conter ou fazer-se acompanhar por este aviso.

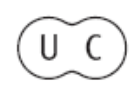




\section{CONIMBRIGA}

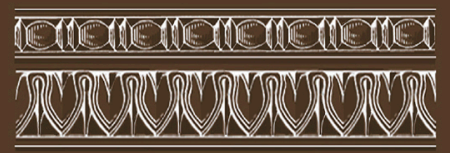

INSTITUTO DE ARQUEOLOGIA

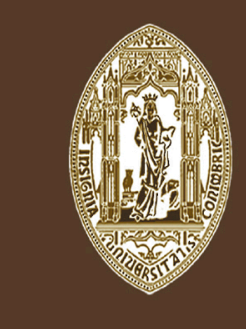

VOLUME XLVII - 2008

FACULDADE DE LETRAS UNIVERSIDADE DE COIMBRA 


\section{Blanca María PrósPer}

Profesora titular de lingüística indoeuropea universidad de Salamanca

Dpto. de filología clásica e indoeuropeo.

Pl. Anaya, S/N

37001 Salamanca

«LOS NOMBRES 'ITÁLICOS' DE LOS ASTURES MERIDIONALES» "Conimbriga" XLVII (2008) p. 145-169

RESUMEN: Este artículo está dedicado a tres importantes etnónimos astures, los ZoElaE y sus dos subdivisiones conocidas hasta ahora, los Desonci y los Tridiavi. Un examen etimológico detallado de estos nombres los relaciona directamente con las lenguas itálicas y no, como suele suponerse, con las celtas.

ABSTRACT: This paper aims to clarify some Asturian ethnonyms which have remained hitherto inadequately understood. These are the ZOELAE and two tribes belonging to this group, the Desonci and the Tridiavi. A thorough etymological approach reveals a very ancient lexical affinity of these names with the Italic languages. 
(Página deixada propositadamente em branco) 


\section{«LOS NOMBRES 'ITÁLICOS' DE LOS ASTURES MERIDIONALES»}

\section{El nombre de los Zoelae y el nombre de los Julios}

El creciente interés por las lenguas de los pobladores occidentales de la Península Ibérica, celtas y no-celtas, ha dado lugar en los últimos años a numerosos estudios, tanto de detalle como, por vez primera, globales. Además de una mejor comprensión general de la naturaleza y funciones de los teónimos indígenas, toda esta obra ha tenido como resultado un considerable refinamiento interpretativo de la fonética y la morfología de las lenguas en cuestión. Un pequeño problema, que no obstante ha dado lugar a un cierto debate internacional en los últimos tiempos, es el del resultado del segmento indoeuropeo $* d y$ - en el dialecto o conjunto de dialectos que yo por comodidad englobo bajo la denominación de lusitano-galaico (cf. LRP, passim). Todo esto ha sucedido al hilo de la interpretación de una de las divinidades más repetidas del panteón occidental: REVE.

Algunos autores han sugerido que se puede identificar REVE, una divinidad bien documentada tanto en la Lusitania Emeritense como más al Norte, en Galicia, con el dativo del nombre de la divinidad celeste de los indoeuropeos por antonomasia, *dyēu-s. Así lo ha creído C. Búa (1997). Algo más recientemente, un estudioso polaco, K. T. Witczak (1999), propone de nuevo una identificación entre REVE y $* d y \bar{e} u-s$. Se trataría de una generalización del grado pleno a todo el paradigma. Por tanto, la protoforma de REVE sería * dyew-ei (véase el paralelo de osco DIÚVEÍ). El principal argumento lingüístico que esgrime el autor a favor de esta idea es el cambio fonético /d/ $>/ \mathrm{r} /$, que cree poder encontrar en varias otras formas, supuestamente con /d/ etimológica, a todo lo largo de la cornisa occidental de Hispania, y que compara con el cambio fonético umbro /d/ > /r/. Hay que notar, sin embargo, que éste no se produce precisamente en posición inicial sino, en general, en posición 
intervocálica y a veces ante alguna consonante, contextos en que el rotacismo puede explicarse a partir de un proceso fónico de lenición y asibilación $*-\mathrm{V} d \mathrm{~V}->*-\mathrm{V} \partial \mathrm{V}->-\mathrm{V} z \mathrm{~V}-$, y que en concreto un cambio $* d y \mathrm{~V}$ $>*_{r} \mathrm{~V}$-, que es el verdaderamente requerido por la etimología de Witczak, queda inexplicado. Por lo demás, en umbro los resultados de /d/ y $/ \mathrm{r} / \mathrm{o} / \mathrm{s} /$ intervocálica son claramente diferentes y se grafían consiguientemente de forma diferente, como se desprende de peřum/Perso "suelo" (<*pedom), frente a aferum/FEROM "llevar" (<*bher-) y la forma rotatizada de la marca morfológica de genitivo de plural femenino en -aru $\left(<*_{-}-\overline{a s o m}\right){ }^{1}$

Con esto se ignoraba sumariamente un trabajo aparecido algo antes. La interpretación de los epítetos como nombres de ríos, y, con ella, la certeza de que debe considerarse REVE como una palabra para "río", había sido ya señalada por F. Villar en 1996,2 partiendo de un minucioso estudio etimológico, y se ve incontestablemente confirmada hoy por la subsiguiente aparición de un texto inscrito en el mausoleo de un sevir augustalis emeritense. ${ }^{3}$ La iconografía del monumento, donde se representa al ANA como un viejo y al BARRAECA como un joven, provistos de cuernos de la abundancia, deja poco lugar a dudas sobre el referente fluvial de estos dos nombres, que como ya viera Villar no aluden a otra cosa que al río Guadiana y a uno de sus afluentes, el Albarregas, cuyos nombres se nos han transmitido, obviamente, a través de los árabes.

${ }^{1}$ Por otra parte, la explicación de las excepciones a esa regla /d/ $>/ \mathrm{r} /$, basada en que se trata en realidad de casos de la antigua aspirada /dh/, como en lusitano DoENTI, me resulta increíble a la vista de que, al menos para la labial aspirada contamos con ejemplos de fricativización y ensordecimiento /bh/ > /f/, como en IFADEM $(<*$ en-bhatyo-). Cf. B. Ma Prósper (2004).

${ }^{2}$ Con la bibliografía anterior. Véase también el resumen bibliográfico de $D I P, \mathrm{pp}$. 263-68. La idea de que se trataba de un dios-río ya había sido sugerida por F. Fita sobre la identificación de REVE con lat. rīvus, que ya no parece morfológicamente viable, sin embargo.

${ }^{3}$ La aparición conjunta de ambos elementos en la inscripción principal del mausoleo de un sevir augustal, Iulius Successianus, condujo a A. Canto (1997) a sugerir que este culto debía estar vinculado con las funciones habituales de un augustal y, por tanto, con el culto imperial. El ejemplo emeritense confirma que el culto imperial aprovechaba en Hispania la preexistencia de núcleos de cultos indígenas, y en este caso de cultos a las confluencias. Tal asimilación es conocida en el santuario confederal galo de Lugdunum en la confluencia entre el Ródano y el Saona, situado frente a Condate, donde hubo históricamente un altar dedicado al emperador Augusto. 
No obstante lo terminante de esta constatación, otro conocido estudioso polaco, W. Mańczak (2006), ha remachado de nuevo muy recientemente la hipótesis de que REVE proviene de indoeuropeo *dyeu-, con el argumento, ya empleado por él mismo en numerosos trabajos, de que aquí se ha producido un cambio fonético irregular debido a la alta frecuencia de uso de esta forma. El autor sustenta esta hipótesis en el gran número de veces que el nombre mismo de "Dios" experimenta evoluciones no esperadas (a menudo, eso sí, porque entra en la formación de compuestos, con lo cual no son propiamente inesperadas, por mucho que su dirección sea menos previsible). Su argumento deja de ser comprensible, por otra parte, cuando trae a colación el nombre latino Iuppiter (de un vocativo * dieu pater), del que dice que es, de entre miles de palabras que empiezan por $d$, la única que ha perdido la dental inicial, debido, evidentemente, a que esta palabra, que designaba al dios más importante del panteón romano, era muy frecuentemente utilizada.

Esta afirmación no dejará de resultar algo rara a cualquier lector especializado, puesto que de ninguna manera puede compararse la evolución de una consonante inicial ante vocal con la correspondiente evolución en los grupos $* d y \mathrm{~V}$ - y $* d w \mathrm{~V}$-. Resulta que la simplificación $* d y$ $>y$-, tardía como parece, es al menos tendencia común a todas las lenguas itálicas, que muestran un abanico de resultados: en alfabeto nacional, $\langle i>-,<d i>$-, en alfabeto latino, $<\mathrm{I}>$-, en alfabeto griego, $\langle i>-,<\delta i>$. $\mathrm{O}$ sea, que en todas ellas se habría producido este fenómeno irregular, en la misma dirección, y sin embargo de forma independiente. Que en el caso lusitano se haya dado el paso, pretendidamente irregular, $d->r$ es una conclusión que depende de la hipótesis misma de que esa etimología es la correcta, hipótesis que carece de apoyo externo, y que además ignora esa misma consideración anterior: No es apriorísticamente comparable la evolución de $d$ - con la de $d y$-. Y el hecho de que formas diversas de esta misma raíz hayan evolucionado en latín en la misma dirección, cuando no había ya posibilidad de que se identificaran con el teónimo, ni siquiera de que se analizaran correctamente, es igualmente revelador: Véase el ejemplo de iubar "estrella matutina", que los especialistas hacen proceder de un compuesto posesivo indoeuropeo $* d y u$ bheHzes "que porta la claridad del día".

Abundando en lo que ya dije en trabajos anteriores, aquí quiero volver a refutar la idea de que /d/ evoluciona a /r/ en Hispania basándome en una nueva interpretación de un nombre muy conocido, el etnónimo Zoela. Los Zoelae ocupaban una zona de amplitud ignota que se 
suele situar entre Zamora y la región portuguesa de Tras-os-Montes, en los alrededores de Braganza. Son mencionados como populus del convento astur por Plinio (Nat. Hist. 3, 28). Pero han pasado a la historia fundamentalmente como protagonistas de un pacto que se ratificaba periódicamente, y que se nos ha conservado a través de dos documentos, el primero de los cuales es la famosa Tabula de Astorga, del año 152 d.C., cuya primera parte refleja un pacto que data del año 27 d.C. (CIL II, 2633). ${ }^{4}$

Por lo demás, estos Zoelae son conocidos por algunas inscripciones funerarias, como PAEDATVRA / T(ITI) I(VLI?) VALENTIS / ZoELAE (CIL II, 2651), o votivas, como DeO / AERno / ORdo / ZoElAR(VM) / EX vото (Braganza, Castro de Avelãs = CIL II, 2606).

Como sabemos por la primera parte de la Tabula de Astorga, este pueblo, que pertenecía a la rama de los Astures Augustan̄, conocía originalmente una división en dos etnias o gentilitates de inferior rango, los Tridiavī y los Desoncī. En la segunda parte, que data de 152 d.C., fecha de confección de la tábula misma, ya se mencionan varios grupos más como gentes que, junto a la gens misma de los Zoelae con sus dos subdivisiones mencionadas, constituyen una unidad política superior del mismo nombre en consonancia con las progresivas exigencias de la administración romana.

Todo esto ha dado lugar a una fructífera polémica entre los historiadores sobre la naturaleza respectiva de gens y gentilitas como unidades de adscripción parental o étnica del individuo, sobre los que pueden consultarse por extenso los trabajos de $\mathrm{M}^{\mathrm{a}} \mathrm{C}$. González Rodríguez. Como indica esta autora (1997, pp. 70 y ss.), la gens Zoelarum debía de constituir una comunidad política radicada en Curunda. Los miembros de las gentilitates de los Tridiavī y Desoncī estaban seguramente unidos

${ }^{4} \mathrm{El}$ texto de la primera parte del pacto, en los pasajes que nos ocupan, dice:

[...] GENTILITAS DESONCORVM EX GENTE ZOELARVM ET GENTILITAS TRIDIAVORVM EX GENTE IDEM ZOELARVM HOSPITIUM VETVSTVM ANTIQVOM RENOVAVERVNT [...] EGERVNT ARAVS ABLECAENI ET TVRAIVS CLOVTI DOCIVS ELAESI MAGILO CLOVTI BODECIVS BVRRALI ELAESVS CLVTAMI PER ABIENVM PENTILI MAGISTRATVM ZOELARVM ACTVM CVRVNDA.

Y su segunda parte:

[...] GENTILITAS DESONCORVM ET GENTILITAS TRIDIAVORVM IN EANDEM CLIENTELAM EADEM FOEDERA RECEPERVNT EX GENTE AVOLGIGORVM SEMPRONIVM PERPETVVM ORNIACVM ET EX GENTE VISALIGORVM ANTONIVM ARQVIVM ET EX GENTE CABRVAGENIGORVM FLAVIVM FRONTONEM ZOELAS [...]

Conimbriga, 47 (2008) 145-169 
por vínculos de parentesco que se remontan seguramente a más de un siglo atrás, ya bastante laxos con el paso de las generaciones y, posiblemente, por adicionales vínculos de tipo territorial.

En nuestro epígrafe me parece por consiguiente probable que las entidades denominadas gentilitates no tengan que ver directamente con los grupos de parentesco bien conocidos en el mundo celtibérico, que aparecen en genitivo de plural dependiendo de un antropónimo y que a su vez son morfológicamente analizables como antropónimo, supuestamente el nombre de un antepasado cercano, como un bisabuelo, y un sufijo velar, habitualmente ${ }^{*}-i k o-$, que señala la relación entre este antepasado y el grupo de sus descendientes. Como señala González Rodríguez, este tipo de organización es relativamente infrecuente entre los astures, frente a lo que sucede con sus vecinos, los cántabros vadinienses, y geográficamente más oriental. Como vamos a ver, no obstante, éste no es el caso en lo referente a la segunda parte del pacto.

Como es bien sabido, sólo existe un caso más de aparición de la palabra gentilitas en la epigrafía hispana: En un epígrafe de territorio vetón de Oliva de Plasencia (Cáceres) se conserva una dedicación a unos Dit Lares Gentilitatis CAPETICORVM. Es éste uno de los escasísimos ejemplos en que los dioses lares, que encubren sin duda divinidades protectoras indígenas asimiladas a aquellos, aparecen protegiendo a un grupo humano y no a una localidad o territorio. Nada sabemos de la procedencia del dedicante, que no nos ha dejado ni su nombre.

No sólo no se documenta un antropónimo que pueda haber proporcionado la base de CAPETICORVM, sino que esta forma requiere un análisis incompatible con la celticidad: *kap-et- se puede interpretar sin problemas como un nombre de agente de la misma raíz que el verbo latino capio, y que significa literalmente "que coge", y puede entenderse en el sentido bélico de "devastador" o quizás en el puramente territorial de "que abarca, que se extiende". Podría ser incluso un correlato exacto de la forma conservada en antiguo indio en una forma de la que solamente sobrevive el dual aislado kapatī "recipiente". Naturalmente, cabe siempre contraargumentar que se trata de un nombre de agente celta *gab-et-, en este caso con la azarosa representación hipercorrecta $<\mathrm{P}>$ por $<\mathrm{B}>$. Aunque así fuera, y es peligroso llegar a esta suerte de conclusiones sobre la base de un ejemplo aislado, subsiste el primer problema. En conjunto, cabe sospechar que se trata, como en el caso de Tridiavī y Desoncī, de una denominación grupal no relacionada direc- 
tamente con las organizaciones familiares o clanes, propias del mundo celta hispano y en concreto de Celtiberia. ${ }^{5}$

Por otro lado, no deja de llamar la atención que los cuatro grupos adicionales mencionados en la segunda parte del pacto sean, precisamente, adjetivos sufijados en -iko-: Se habla de individuos ex gente Avolgigorum, ex gente Cabruagenigorum, ex gente Visaligorum. Es típico de los astures, precisamente, el empleo de la fórmula onomástica tanto con genitivo de plural (Alia Verna LegirnicCorvm, Astorga, León) como con ex gente + genitivo de plural (Ex GENTE ABILICORVM, Morcín, Asturias). Por lo tanto, es posible que en la segunda parte del pacto la forma gens se emplee ya de forma superflua en referencia a la organización familiar típicamente celta, como probablemente pasa con las cinco inscripciones astures septentrionales de este tipo. Curiosamente, estas tres formas se relacionan sin problemas con documentación celta bien conocida: Avolgigorvm, que además tiene un pendant cántabro AvLGigVN, puede derivarse de un *awol-iko-, a su vez derivado de *awo-lo-, un diminutivo "nietecito" que existe en la antroponimia de las Galias en la forma Avolvs. Visaligorvm procede de *wiss-alo-, derivado de *wid-tu- "sabiduría", y aparece en versión puramente indígena en el genitivo de plural uisalikum del bronce de Botorrita III. Por último, CABRVAGENIGORVM puede hacerse proceder de un compuesto de creación tardía *gabro-adgeno- "perteneciente al clan de la cabra".

Por lo demás, es posible que exista una relación con la forma del epíteto de una divinidad de lectura e interpretación difíciles, hallada en la provincia de Zamora (Vigo de Sanabria, Galende): Según los editores dice MADARSSV + BlACAV. ${ }^{6}$ En un trabajo de R. García Rozas y J. A. Abásolo ${ }^{7}$ se edita también esta ara, pero se interpreta el teónimo como Madarsso / SoElagav(M). Una lectura parecida es la que ofrece A. Redentor (2006, p. 261), que opta por MADARssv SoElagav, e indica, entre otras posibilidades, que el origen último de este nombre puede relacionarse etimológicamente con el de los Zoelae. Por el momento me parece que hay que tomar con cautela esta posibilidad por diversas razones: El epíteto se desvía claramente de la tipología normal

5 A partir de ahora y para evitar confusiones denominaré simplemente "nombres de familia" a estos grupos.

${ }^{6}$ Cf. J. M M Bragado Toranzo - S. Ma García Martínez (1997).

${ }^{7}$ Que permanece en el día de hoy en prensa, aunque en realidad es anterior. 
en que no es habitual una derivación en -kawo- y en que no es típico, aunque tampoco imposible, que el epíteto divino sufijado se relacione con un etnónimo, a menos que ambas cosas puedan derivarse, paralela y por tanto independientemente, de una misma raíz o de un mismo elemento del vocabulario apelativo. Como voy a intentar desarrollar a continuación, pienso que esto no es así en el caso que nos ocupa, y que Zoelae era una forma ya probablemente inanalizable para el hablante y pertenenciente en exclusiva al campo de la onomástica.

En una reciente reunión científica, ${ }^{8}$ J. L. García Alonso trae a colación una hipótesis de P.-Y. Lambert (1980, p. 177), que retrotrae una serie de nombres propios celtas a *so-welo- (que hay que corregir en *su-welo-, como él mismo indica personalmente a J. L. García Alonso, cit., n. 4), forma que podría querer decir "que ve bien" y que tiene la ventaja de aproximarse a onomástica de tipo personal celta insular y bretona, como abret. Hoel y galés Hywel. ${ }^{9}$ Pero éste nota también correctamente que cualquier etimología basada en la presencia de $s$-inicial requiere suponer que ésta ha sonorizado en un contexto poco propicio para ello. Pero podemos añadir todavía otra objeción: Aunque el empleo de esta grafía se base en una peculiar adaptación del alfabeto latino a la expresión de diferencias (¿fonológicas?) perceptibles para los propios indígenas, el empleo de un grafo $<\mathrm{Z}>$, ya de por sí infrecuente en latín, para la notación de [z], tiene paralelos muy poco firmes en nombres celtas documentados en inscripciones en alfabeto latino.

$A C S$, fuente secundaria de gran utilidad, pero que se revela en este punto más promiscua que nunca, ofrece en efecto varios casos de empleo del grafo $<z>-$, y concretamente en posición inicial. Pero, aparte de que a menudo no menciona las fuentes (que en todo caso son casi siempre medievales) ni indica siquiera que lo que da como lemas son en realidad en muchas ocasiones formas reconstruidas, se observa que los

8 Celtic and its Neighbouring Languages, Salamanca, mayo de 2006. Cf. J. L. García Alonso (2007, en prensa).

${ }^{9}$ Lambert se pregunta si ha habido un estadio /u/ > /o/. en realidad esto no supone ningún problema en sí, dado que $\mathrm{K}$. McCone ha demostrado de forma convincente que se ha dado un cambio fonético proto-celta $/ \mathrm{u} />/ \mathrm{o} /$ en contacto con $/ \mathrm{w} /$, a su vez seguida de cualquier vocal a excepción de /i/. El único obstáculo aparente a esta identificación consiste en que exige que el compuesto haya dejado de ser inteligible ya desde fecha de unidad celta, o de lo contrario su primer elemento habría recuperado su forma $s u$ - sobre el modelo de tantos otros. 
únicos ejemplos donde la etimología es claramente /s/ están, en realidad, en alfabeto griego, donde en época tardía era normal reproducir una silbante sonora por medio de $\langle\xi\rangle$. En realidad, lo que sí sabemos a partir de textos epigráficos y no epigráficos es que en latín tardío el grafo $<\mathrm{z}>$ tenía un valor de dental sonora palatalizada [dj] y luego más probablemente de dental africada [dz], que permitía la aparición de dobletes gráficos, como zebus por diebus e hipercorrecciones como baptidiare por baptizare. ${ }^{10}$ Todo lo cual indica que si en la zona de los astures al menos se hizo un uso semejante de este grafo, la etimología difícilmente puede tener una silbante inicial, sonora o no. La prueba patente de que no estamos ante un uso idiosincrático por parte de los indígenas de la grafía latina la tenemos en que Plinio menciona a los Zoelae y el derivado Zoelicum en total dos veces a lo largo de su obra, y sin la menor variación gráfica respecto de los testimonios epigráficos hispánicos.

Por su parte, García Alonso ha defendido la posibilidad de retrotraer el nombre de los Zoelae a una forma inmediatamente anterior *dyoilo-. A su vez, esta forma se explicaría partiendo de la base de que se ha producido una "anticipación de yod" a la sílaba anterior, como la que defiende para otras muchas formas y en diversos trabajos P. de Bernardo Stempel. De modo que habría que partir, en última instancia, de *dyol-yo-. Y a su vez esta forma sería analizable como un compuesto celta donde el prefijo $d \bar{\imath}$ asumiría un valor intensivo: Un plural *Di-ol-ia vendría a significar "those who destroy a lot".

Pues bien, si por una parte no puedo estar de acuerdo con este último fenómeno fonético, por el que el diptongo intermedio -oi- se explica por anticipación de $-y$ - de la sílaba siguiente, ${ }^{11}$ es en cambio cierto que la atribución de la inusual grafía $<z>$ - a un grupo inicial $* d y$ -

10 Cf. W. S. Allen (1991, p. 58). Obsérvese que hay pruebas indirectas de un valor africado en los dialectos griegos de Italia, como se deduce del uso que hace el osco del alfabeto griego en el caso del dativo $\zeta \omega F \eta \iota ~(<* d y e w-e i$, Rossano di Vaglio), frente a ıovFๆı (Paestum), formas que tienen un equivalente directo en alfabeto latino, con empleo precisamente del grafo $\langle Z\rangle$ - para notar una africada [dz] o más probablemente una silbante fricativa sonora [z] del mismo origen, en el genitivo Zoves de la Tabula Bantina $(<*$ dyew-eis).

11 Véase la larga serie de contraejemplos ofrecidos en F. Villar - B. Ma Prósper (2005, cap. VIII), que habría que molestarse algo más en discutir uno por uno, en vez de ignorarlos sumariamente como hace De Bernardo. 
me parece una idea más que sugerente, que abre posibilidades insospechadas. Partiré de que se trata originalmente de un grupo consonántico $* d y$-, y no del resultado secundario de una preposición celta $d \bar{\imath}$. Si así fuera, la frontera de compuesto habría impedido con toda probabilidad que la vocal /i:/ hiciera coalescencia con la dental, porque de esta manera se perdía definitivamente el valor original de esta forma, que entendida así dependería crucialmente de su segmentación. Véase por ejemplo en galo lo que sucede con los compuestos diastu-, diacus.

En realidad, hasta ahora sólo había un ejemplo claro de la evolución de un grupo indoeuropeo $* d y$ - en toda la franja occidental de Hispania: El proporcionado por el nombre de la divinidad IOVEAI, que se documenta en la inscripción de Lamas de Moledo y que difícilmente puede interpretarse como otra cosa que no sea un derivado $*$ dyew-yodel nombre del cielo, *dyeu-, derivado por cierto muy bien documentado en las lenguas itálicas antiguas.

Pues bien, nada impide suponer que, a lo largo de toda la cornisa occidental, se dieron evoluciones diferentes del grupo primitivo $d y$-. Si IOVEAI muestra pérdida de la dental como el latín, hasta ahora atestiguada sólo en la Lusitania Scallabitana, el nombre de los Zoelae testimonia un resultado africado dental sonoro [dz] o bien con menor probabilidad un resultado fricativo sonoro [z], es decir, un resultado "a la griega" en un dialecto no-celta del área meridional del convento astur. ${ }^{12}$

El postulado de la pérdida de /w/ en posición intervocálica no tiene ningún inconveniente fonético y sí muchos paralelos en la misma zona y para este mismo contexto. En $L R P$, cap. XV, yo me planteaba la necesidad de entender varios casos en que se documenta una secuencia $<\mathrm{OE}>$ - como procedentes no, de un diptongo indoeuropeo $*_{-}-\mathrm{oi}_{-}$, sino de un segmento -owé-, donde la pérdida de /w/ se explica en virtud de la pronunciación in allegro. Los ejemplos más notables de una evolución *-Co.w V->-Co.V- son: El antropónimo BoElivs (Bande, Orense), BoEli (Villamesías, Cáceres) procedentes de una forma anterior *bowélius, que a su vez viene de * ${ }^{w}$ ow - "buey"; OILAM (Guarda) de *owílam "oveja", forma conservada tal cual al otro lado del continuum indoeuropeo en sánscrito avilā; ${ }^{13}$ y la forma verbal en $3^{\mathrm{a}}$ pers. pl. de presente DoEnTi (Lamas de Moledo,Viseu), de *dowénti.

12 Algo parecido sucede cuando se comparan diversas formas procedentes de zonas diversas de implantación del osco, como explico en la nota 10 .

13 Cf. K. T. Witczak (2005). 
El nombre de la dinastía Julia se hace proceder del nombre de su fundador mitológico Ascanio Julo, hijo de Eneas, legitimador a posteriori de las aspiraciones de poder de la gens. A su vez, no parece haber ninguna duda de que Iülus como su derivado Iülius proceden del nombre indoeuropeo del cielo y de la divinidad celeste *dyeu-. Ya la obra tardía Origo gentis romanae atribuye al cónsul y autor erudito L. Caesar la opinión de que no sólo Ascanio era de la estirpe de Júpiter, sino que "per diminutionem declinato paululum nomine primo Iolum, postea Iulum appellarunt" $(15,5) .{ }^{14} \mathrm{El}$ sobrenombre Iülus de Ascanio fue tempranamente utilizado por los Julios para reivindicar su origen divino, y no parece que haya sido ajeno a esto el juego de palabras que relaciona a Iülius con la divinidad Vediovis "joven Júpiter". Así, los julios son mencionados por vez primera en una inscripción de un altar del S. II a.C. que reza Vediovei Patrei Genteiles IVliei (CIL I² 1438).

Pero el hecho es que también en la tradición científica se retrotrae Iūlius a una forma itálica *Iouǐlios (por ejemplo en IEW, p. 184; LEW I, p. 729, St. Weinstock 1971, pp. 9-10). Los motivos para esto no resultan del todo evidentes: Parecen basarse, sobre todo, en la existencia de una serie de apariciones oscas, concretamente capuanas, de una palabra iúvilam (ac.sg.), iúvilas (nom. pl.), que designa estelas de arcilla o piedra. Como ya indica J. Untermann (2000, p. 188), la etimología de esta forma difícilmente puede portar un sufijo *-̌̌lo-, puesto que la vocal breve - $i$ - en posición medial se habría sincopado. Por ello, favorece la reconstrucción de una protoforma itálica $*$ dyowyelo-, que hasta ahora parece la más probable entre las diversas que se han propuesto (cf. ibidem). Añadamos que tal secuencia *-řlo- debería entenderse probablemente, a la luz de nuestros actuales conocimientos sobre la formación de palabras del indoeuropeo, no como un sufijo originario, sino como amalgama secundaria de varios sufijos primitivos o como un sufijo tardío surgido por metanálisis. Con esto se destruye en cualquier caso la relación directa de iúvilam, etc., con Iülus, que en cualquier caso tiene una vinculación etimológica obvia, pero que difícilmente puede ser una forma idéntica.

Por lo demás, y a pesar de que la forma Iūlus no se documenta nunca con diptongo, podemos extraer indirectamente de la tradición

14 Puede consultarse el resto de las etimologías transmitidas en la Antigüedad y su valoración como testimonio en J.-Cl. Richard (1986, p. 183). 
más antigua la certeza de que $-\bar{u}$ - procede de la reducción de un segmento más largo, conservado aún en latín primitivo, a través de la noticia de la evolución Iolus > Iulus (supra) y también, como indica Richard (1983, p. 117), a través de la etimología transmitida por Servio (Aen. 1, 267) y atribuida igualmente a la autoridad de L. Caesar, que es "ioßo óv , id est sagittandi peritum".

Epigráficamente se documenta, de acuerdo con los diccionarios, una forma IVILIVs que sólo he podido encontrar en ILAlg 2, 1, 1957, 3380 (Celtianis, Numidia, Argelia) y en una moneda de época augústea datada con posterioridad al 40 a.C., cuyo reverso dice Divos IVILIVs; ambas, como se deja ver, algo tardías para ser de mucha utilidad, además de unas Figvlinae IVILIANAE en CIL XV, 256 y una inscripción arcaica (CIL I, 518).

Ya F. Solmsen (1894, p. 145) hacía proceder ambas formas, esto es, Iǔĭlius y Iuilius, de un mismo étimo *Iouilios, que compara con la relación entre Cloelius, Boelius y sus supuestos étimos *Clǒuľlios, *Bǒuǐlios, a su vez formas derivadas de las que se documentan en Clouius y Bouius. Esto haría algo más plausible, si bien más compleja, la derivación $*$ dyeu- $\rightarrow *$ dyew-yo- $\rightarrow *$ dyewi-lo- $>$ Iülus. Una derivación similar no sería impensable para la forma astur. Es decir, una protoforma hispana $*$ dyewilo- habría evolucionado regularmente a $* d y o$ wilo- y la pérdida de /w/ habría dado como resultado un diptongo secundario -oi- que, naturalmente, tendería a grafiarse como $<\mathrm{OE}>$ siguiendo la costumbre latina, aunque representaciones propiamente indígenas como Oilam (Cabeço das Fráguas), de *owila “oveja”, hacen esta opción menos recomendable.

No obstante, B. Vine (1993, p. 102) incluye convincentemente el caso de IVILIVS en CIL I, 518 entre otros con anticipación gráfica de $<i>$ que entra en un grupo de formas prenestinas y propiamente romanas en que a una vocal larga le sigue $<\mathrm{i}>$ anticipada de la sílaba siguiente, como en CIL I, 552 PAINISCOS y 569 PoimilionOM. Por consiguiente, según este autor, IVILIVs viene a reflejar una secuencia secundaria /Iūilius/. En conclusión, es innecesario contar con este grupo aislado de formas a la hora de establecer el origen último del nombre Iülus.

Examinemos una posibilidad alternativa: Que el nombre Iülus provenga más bien de una forma indoeuropea occidental *dyew-elo- "pequeño o joven dios (celeste)", que es menos problemática desde un punto de vista formacional, y que además es perfectamente posible desde el punto de vista de la evolución fonética. Es cierto que el cambio de 
género resulta problemático en el etnónimo Zoela; pero no mucho más que otras denominaciones étnicas que, siendo antiguos adjetivos derivados o participios, han pasado al género femenino, como los pueblos británicos Karnonakai, Dekantai, Nouantai, o, sin ir más lejos, los Celtae.

Una protoforma *dyew-elo- debió dar regularmente en itálico común *dyowelo-. Observemos pues que sus características son distintivamente itálicas: Generalización del grado pleno *dyew- a todo el paradigma y presencia de un sufijo de diminutivo - elo-. A partir de aquí el segmento -owé- con segunda vocal tónica evoluciona a lo largo de la historia del latín a $-\bar{u}$-, como en rūsticus < *rowestikos, nūntium < *nowentyom. En consecuencia, el nombre de los Zoelae y el nombre de $I \bar{l} l u s$ serían idénticos, apuntalando la relación establecida en $L R P$ entre el dialecto (o los dialectos) del Occidente peninsular y las lenguas itálicas. ${ }^{15}$

La divinidad indígena más claramente asociada a los Zoelae es AERno. Como ya indiqué en otro lugar ( $L R P$, cap. X.II), se documenta con total seguridad tan sólo en tres dedicaciones votivas (ERRB, pp. 46-49, sección II.1, nrs. 1-3), que pertenecen al Norte de Portugal y se hallaron en Castro de Avelãs, sitio arqueológico próximo a Braganza, y Malta (Macedo de Cavaleiros), de modo que, como ya he adelantado, suele considerarse que ésta era la zona de implantación de los Zoelae. En su momento ya defendí la atribución etimológica a un derivado *ayer(i)-no- "luminoso". Por tanto, consideraba que AERNo podía ser el epíteto de una divinidad solar o celeste. A. Redentor ha señalado en un trabajo reciente (2006) que, aparte de AERNO, la divinidad predominante en la zona es Júpiter. ${ }^{16}$

15 G. Devoto (1967, p. 331) relaciona el gentilicio latino Iuvilius con una forma lepóntica Iuvilios. En realidad, una secuencia como ésta sólo puede extraerse de una inscripción procedente de Cernusco Asinario, en el área de Milán. Nadie la ha visto desde 1879, y P. Solinas (1995, p. 364, nr. 98) la transmite como - ?tiusiuilios, sugiriendo que puede segmentarse como -tiu siuilios. En cuyo caso se trataría de una fórmula onomástica bimembre en nominativo, compuesta de un tema en nasal y un apositivo en -ios. En cualquier circunstancia resulta evidente que esta forma es inutilizable para el problema que nos ocupa.

16 En griego, el adjetivo ท’દ́ gen en el locativo *āyer- $i$, tiene tardíamente por homofonía el significado de "aéreo". Un posible equivalente del mismo origen en koiné podría ser la forma ท̉દ́ inscripción votiva de origen minorasiático, y concretamente lidia, que engrosa la nómina de las numerosas advocaciones de Zeus de la epigrafía helenística de la zona. 
En otras palabras, sospecho que, a diferencia de lo que sucede con REVE, el epíteto sin teónimo AERno sí que puede encubrir en realidad una forma local del culto a la divinidad celeste indoeuropea. No es casualidad, por consiguiente, que sus principales devotos, los Zoelae, deriven su nombre de $* d y e u$-. En aquellos tiempos todavía es posible que tuvieran conciencia de su propia vinculación con la divinidad principal del panteón primitivo, y que entendieran aún la relación de su propio nombre con el de ésta.

\section{El nombre de los Tridiavi y los términos latinos de parentesco}

Del etnónimo Tridiavī, gentilitas de los Zoelae, se ha dicho hasta la fecha, a mi conocimiento, que es un posible derivado de indoeuropeo *triti(y)os "tercero". ${ }^{17}$ Entre los cántabros vadinienses, en concreto en una inscripción de Monte Cildá (Palencia, CIL II 6296), se documenta además un nombre DoIDER[VS] TRIDI[A]VM que en principio, y dadas las dificultades de lectura, debería tomarse con cautela, dado que lo más habitual en este grupo son los genitivos de plural dotados de sufijo velar. ${ }^{18}$

A pesar de ciertas opiniones al respecto, por lo demás comprensibles, dada la rareza del caso, el sufijo -awo-/-âwo- deriva nombres a partir de otros nombres, topónimos y antropónimos, pero no se emplea para formar nombres de organización familiar. Cabe pensar, no obstante, que aquí el individuo hace referencia a una unidad superior. Así creo que debe entenderse un supuesto grupo familiar ARAVM en León (CIL II, 5716): Manili Aravm Elani F Va(DiniEnsis). No puede ser casual que en Lusitania esté muy bien documentado el etnónimo Aravī y la civitas Aravorum, y es muy posible que la familia de este ciudadano vadiniense pertenezca o haya pertenecido en último término de este grupo humano. ${ }^{19}$ Dada la coincidencia en el hecho de que ninguno de

${ }^{17}$ Cf. por ejemplo A. Tovar (1954), y más recientemente Ma L. Albertos Firmat (1985) y J. Ma Vallejo Ruiz (2005, p. 547).

18 Las excepciones, pocas, parecen deberse a errores o a malas lecturas, como Pentiorv(M) por Pentiocvm y OILARIdvn por OILARIQvn en Villaviciosa (Asturias). Véase algo más abajo lo que digo a propósito de la expresión Ex GENTE PEMBELORVM.

${ }^{19} \mathrm{El}$ nombre de los Aravì tiene correspondencias celtas extrahispanas, por ejemplo en los Araviscī o Eraviscī de Panonia. En mi opinión no es un compuesto ni un deri- 
estos dos casos conoce la forma típica de los grupos familiares con sufijo velar y en que ambos son conocidos como unidades étnicas de rango superior a las anteriores, es posible formular la hipótesis de que en el nombre de los cántabros Manilius y Doiderus se hace referencia a la vinculación étnica más antigua de su familia, desplazada desde una zona más meridional, donde precisamente el empleo del sistema filiativo de los genitivos de plural era infrecuente.

Sin embargo, es probable que la realidad original sea bastante más compleja. Aún conservamos noticia de los nombres que daban los romanos a las sucesivas generaciones de antepasados, contadas hacia atrás. Figuran ya por su orden en Plauto (Persa 57): pater, auos, proauos, abauos, atauos, tritauos.

Aquí nos interesa el término tritauos. Se trata, en términos actuales, del abuelo del tatarabuelo, es decir, la sexta generación a partir del presente. Se reitera este mismo orden en San Isidoro (IX, 5, 9), para el cual "tritauus ultimum cognationis nomen est".

Curiosamente, la opinión tradicional ha visto en trìtauus una falsa forma latina, no histórica, sino analógica de la correspondiente griega. Por ejemplo, para É. Benveniste (1965, p. 8), trītauus tiene como modelo la forma griega sinónima $\tau \rho \hat{́} \pi \alpha \pi \tau o \varsigma$. Sólo O. Szemerényi (1989, pp. 41-42) ha notado correctamente que hay que entender la forma trìtauus como puramente latina, y que tiene en su base el numeral ordinal *triti(y)os, regularmente conservado en latín tertius. Trītauus procede por tanto en última instancia de *triti(y)o-awo-, compuesto que significa "el tercer abuelo" y que debe su formación a que el antepasado en cuestión ocupa precisamente el sexto lugar desde el presente, con lo cual se entiende que si cada dos generaciones culminan en un abuelo, el sexto lugar lo ocupa un tercer abuelo. Desde el punto de vista de la evolución fonética, tendríamos que contar con una secuencia cronológica *triti(y)o-awo- > *tritiewo- > *tritīwo- (por la ley de la apofonía) $>*$ trītiwo- (por metátesis, lo que explicaría la medida larga en Plauto) $>*$ trītawo- (por refección a partir del simple auus).

Resulta ahora sencillo establecer la relación etimológica con la etnia de los Tridiavī. Sencillamente este nombre mantiene la estructura

vado en -awo-, sino que puede remontarse a celta común *erawo-, luego *arawo- por la acción de la Ley de Joseph, y yendo más atrás en el tiempo, a indoeuropeo ${ }^{*} H_{1}$ erHwo- "tranquilo". El antropónimo Arauus es claramente de idéntico origen, pero puede ser históricamente (al menos parcialmente) independiente del etnónimo.

Conimbriga, 47 (2008) 145-169 
original tal como ha sido reconstruida por Szemerényi para la forma latina trītauus. Es decir, procede directamente de *trity $(o)$-awo-, con ulterior sonorización del grupo $-t y->-d y$-, típica en las consonantes intervocálicas en toda la franja occidental de la Península Ibérica y entre los cántabros. -awo- no es aquí un sufijo formador de antropónimos, sino el segundo término de un compuesto muy arcaico. Estamos, por consiguiente, ante una segunda coincidencia no trivial entre el grupo itálico y los etnónimos de los astures. Que el esquema compositivo es

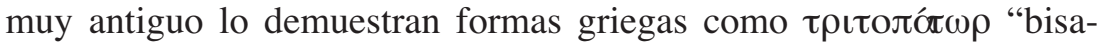
buelo", que se emplea en plural en Atenas para referirse a divinidades ancestrales, y de la cual en ocasiones también se ha considerado que latín trītauus no era más que un calco (cf. LEW II, s.u.).

En galo, es cierto, se conservan algunas formas que ocasionalmente se han hecho proceder de compuestos, y que son probablemente formaciones de fecha dialectal, como VesvaVvs de *wesu-awos, Comavvs de *kom-awos (cf. DLG, s.u. aua). En ellos, *awos quiere decir "descendiente", que es el significado heredado en celta común. Se diría que en el caso de la forma astur estamos ante un compuesto de tipo posesivo, es decir que se entiende por Tridiavī a aquellos que comparten un antepasado lejano de la sexta generación. Naturalmente el significado literal de la forma perdería su sentido con la sucesión de las generaciones, quedando sólo su núcleo: la vinculación con un ancestro común.

No está excluido que, después de todo, se trate de una forma celta, por más que la falta de paralelos hace este extremo totalmente indemostrable: Estaríamos ante la noción inversa de un "sexto descendiente". Esta idea de un "sexto descendiente" se da igualmente en latín, bajo la forma trinepos. Entonces estaríamos en efecto ante unos descendientes de sexta generación, $\mathrm{y}$, dada la identidad formacional última con la forma latina, tendríamos una isoglosa formacional italo-celta de carácter no trivial. La verosimilitud de todo esto, no obstante, se ve algo mermada porque se requeriría que, a pesar de haber quedado desmantelado este sistema de clasificación parental y haber cambiado la forma *awos de significado desde época de comunidad celta, hubiera sobrevivido aislada esta única forma, pero analizada ya y empleada de acuerdo con el nuevo valor de "descendiente" adoptado por la forma simple.

Yendo algo más lejos, podría pensarse incluso que estamos ante un arcaísmo de fecha indoeuropea. La evidencia es claramente escasa: El único dato que puedo aducir para apuntalar esta posibilidad es el nom- 
bre propio licio Epñxuxa, ${ }^{20}$ cuya segunda parte -xuxa es idéntica a lat. auus y a het. huhhas, y cuya primera parte, presumiblemente procedente de *opem, es un adverbio que se cree que significa "después". Podríamos especular con la posibilidad de que se trate de un "abuelo posterior", es decir, el siguiente en la jerarquía, pero contando lógicamente hacia atrás, después del abuelo: es decir, el "bis-abuelo", en latín el proauus.

Por último, una palabra o dos acerca del numeral "tercero" en celta. La única rama celta que conserva como tal un numeral *tritiyo- es el britónico. En celta continental la forma antigua parece más bien *trito-, a juzgar por galo TRITOGENVS, celtibérico tirtu, tirtobolokum. En Hispania, no encontramos en términos generales, con la posible excepción de un antropónimo TriTIAnvs de Salamanca, ${ }^{21}$ formas derivadas de *tritiyo-, sino precisamente de *trito-. El derivado principal de *trito- es precisamente *trit-yo-, común en toponimia y en antroponimia. Y otras frecuentes son tirtouios, tirtanos y los nombres de familia derivados Tritecv(M), tirtanokum, Tritalicvm, TIRTAliQ(VM), TridoNIECVM. Finalmente, los escasísimos casos de derivación en -awo- hispanos tienen como base sustantivos: los lemavī galaicos, el teónimo Colvav (Cáceres) y tal vez el teónimo SANnOAva (Pontevedra), que presentan problemas propios (cf. LRP, caps. VI.III y V.XI).

Por consiguiente, para defender que, después de todo, TRIDIAVORVM y TRIDIAVM portan un sufijo -awo-, habría que aceptar que son etnónimos, pero originados en realidad (lo que tampoco es estadísticamente tan frecuente) en localicios, es decir que estaríamos ante el derivado de un topónimo Tritium. Esto a su vez conlleva problemas menores: El localicio típico porta en este caso, como de costumbre, sufijo velar, como en el epíteto divino TRITIAECio (Cáceres) o la ceca berona Titiakos, Titiako (A.58). Y entre las localidades del área astur y cántabra no figura ningún Tritium, a diferencia de lo que se deduce de los datos de Cáceres y del área nororiental de España. En términos absolutos, pero sobre todo en comparación con la situación de las Galias, la sufijación por medio de -awo-/-âwo- es sumamente infrecuente en la Península, donde, en antropónimos, se documenta más bien un sufijo

20 Cf. por ejemplo H. C. Melchert (1994, p. 307, etc.).

21 Que en realidad imita el esquema de los cognomina latinos Domitianus, Titia$n u s$, etc. La mayor parte de los nombres hispanos en -ianus son interpretables como formas latinas. 
complejo *-aw-yo-. Todo esto hace pensar que el nombre de los Tridiavi $\bar{\imath}$ encubre una realidad diferente y más arcaica, probablemente no celta, y que no se deja explicar por el empleo de un sufijo poco productivo sobre el derivado primario de un numeral.

\section{El nombre de los Desonci.}

Vamos ahora a analizar el nombre de los Desoncī, el segundo gran grupo de los Zoelae según la primera parte conservada del pacto de Astorga. Parece obvio que estamos ante un derivado en *-on-iko- de un adjetivo *dekso- o tal vez de *deks-wo- "derecho, recto". El adjetivo básico, que ha dado lugar a diferentes derivados, es pan-indoeuropeo y procede de la tematización de un tema en silbante *dekos- "lo que está bien, lo que se debe hacer", de manera que nada más puede extraerse sobre su filiación dialectal. En la Península Ibérica tenemos varios nombres de persona de la misma etimología, atribuidos tradicionalmente en virtud de su distribución geográfica a la rama celta: Así por ejemplo Dessvaeona (Burgos) o el topónimo Dessobriga (It. Ant.449, 4), igualmente en Burgos, y probablemente Deixibre (Carbia, Pontevedra), Deixebre (Oroso, La Coruña).

Hay que añadir un dato que tampoco es trivial para la consideración de Desoncorum como etnónimo: Cuando se deriva un nombre de familia de un antropónimo por medio de un sufijo velar, la relación entre la base y el sufijo -iko-, -osko-, -aiko-, suele ser, en la mayoría de los casos, sincrónicamente transparente. Es decir, que estos nombres de familia pueden con toda facilidad descomponerse en unidades discretas. Si se echa una ojeada a la lista actualizada de unidades familiares documentadas que ofrece en sus páginas finales $B B . I V$, se observará que no existe una sola que muestre un final $-<$ onkum $>$ ni $-<$ ONCORVM $>$. J. $\mathrm{M}^{\mathrm{a}}$ Vallejo (2005, p. 287) aporta tan sólo un CoILIONQ(vM) de Salamanca.22

22 Es muy chocante por tanto el contraste con los numerosísimos casos de una secuencia $-<$ ankum $>,-<$ ANCVM $>,-<$ ANCORVM $>$, que paralelamente habría que interpretar como sincopada a partir de *-an-iko-. Como sugirió ingeniosamente J. F. Eska (1995, p. 76), los temas en nasal tienden a emplear en celtibérico el sufijo *-ko-, en vez de sus variantes *-iko-, *-āko-: cf. Aiankum vs Aiu (y EbVRo vs EbVRAnCo, Avo vs Avvancvm, etc.). La Cognatio Magilancvm del bronce de Montealegre se ha visto a veces como resultado secundario de síncopa de la forma MAGILANICVM. Sin embargo, 
Naturalmente, en DESONCORVM es fácil deducir que -<ONC $>$ - proviene de una secuencia -on-iko- que ha sufrido sincopación. Pero, si hubiera un antropónimo celta *dexsonos del que se derivase este nombre, es probable que se actualizara en cada generación la forma derivada correspondiente *dexsoniko-. En nuestro caso la sincopación es muy antigua (ya está presente en la primera parte del documento) y la relación del derivado con la base empieza a hacerse incomprensible. Porque el nombre de los Desoncī es un etnónimo, y en consecuencia no tiene como base un antropónimo. Por lo tanto, sincrónicamente no es analizado como tal (posiblemente ni siquiera se entiende) y su antepasada con sufijo -iko- no se reconstituye mecánicamente con el sucederse de las generaciones.

A manera de conclusión podemos decir que, como ya indica $\mathrm{M}^{\mathrm{a}} \mathrm{C}$. González Rodríguez, la composición de la sociedad astur era un tanto heterogénea. En el caso de los Astures Augustani, la parte primera del documento de Astorga es preciosa para descubrir la existencia de realidades étnicas y/o políticas de diferente rango, lo que aquí se traduce como la gens y la gentilitas, que en mi opinión, fundada en exclusiva en el análisis lingüístico, conserva un componente no-celta, que se remonta a fecha muy arcaica y que tiene grandes similitudes con el mundo itálico. La situación ha cambiado radicalmente en dos siglos, de manera que en la segunda parte, reunidas bajo la expresión Ex GENTE, se tratan como gentes realidades que en mi opinión corresponden simplemente a los genitivos de plural sin mención del nombre que designa ese determinado grupo humano que llamamos organización familiar, 23 como sucede en la zona septentrional, entre cántabros y astures, en las fórmulas onomásticas de los epitafios. Tanto este uso en sí mismo, como el análisis lingüístico de los nombres de las gentes mencionadas remiten claramente al mundo celta aledaño, y pudo tener que ver con la reorganización secundaria de las unidades políticas por parte de los romanos

está bien documentado el antropónimo latinizado Magilo(n)-, de manera que es plausible que su derivado antiguo fuese *magil-n-ko-. MAGILANICVM se explica precisamente por aspiración a la transparencia. Si en cambio la forma más antigua fuera MAGILANICVM, la relación con Magilo(n)- queda sin explicación derivacional, porque lo esperable en todo caso sería *Magilūnikum o la variante latinizada *Magilōnikum.

$23 \mathrm{O}$ bien, ocasionalmente, genitivos de singular, o incluso nominativo o dativo del nombre de familia en concordancia con el antropónimo, como sugiere M. Ramírez Sánchez (2001). 
y su integración progresiva de los Astures Transmontani en la realidad urbana más meridional, o con la absorción de inmigrantes procedentes de la meseta. Se observa fuera de toda duda que los signatarios de la primera parte del pacto, cuya fórmula filiativa es la más sencilla de antropónimo + nombre del padre en genitivo, tienen a menudo nombres celtas, como Magilo Clouti (cf. nota 4). Es posible que pueda decirse otro tanto de la celticidad de los nombres de pueblos astures septentrionales, como Gigurrī y Susarrī. Pero éste será ya el tema de otro trabajo.

En consecuencia, no estoy del todo de acuerdo en que el empleo de fórmulas diferentes (gens, gentilitas, cognatio, genitivos de plural directamente dependientes del antropónimo) haga necesariamente referencia a realidades distintas, como tampoco creo, al contrario, que estas fórmulas fueran sistemáticamente intercambiables, como opina F. Beltrán: El reiterado empleo que hace la tabula de Astorga del término gentilitas, que, casi dos siglos después de la primera consignación del pacto, aparece en la segunda parte del documento en clara oposición al uso de Ex GENTE, hace en mi opinión imposible entenderlo como equivalente del término omitido en las expresiones filiativas con genitivo de plural. Es posible que en ese momento, aparte de civitas u ordo Zoelarum, se siguiera diciendo gens Zoelarum y gens Orniacorum, al igual que gens Asturum. Como indica P. Rodríguez (1994), gens hace referencia extensional al conjunto de personas que integran un grupo, pero se emplea tanto para grupos mayores como para sus subdivisiones. El empleo de gens para grandes grupos y para explicitar la relación entre los antropónimos y los genitivos de plural habría introducido entonces cierta confusión de emplearse en un mismo documento. De ahí posiblemente también la oposición entre gens y gentilitas, entendida como gens de rango comparativamente menor, oposición orientada a hacer más evidente la relación de subordinación jerárquica entre los grupos.

A propósito de las incógnitas que plantea el uso de la expresión Ex GENTE, me gustaría añadir que, además del uso típico astur y en ocasiones cántabro como equivalente de los genitivos de plural, por una parte, y del uso genérico para referirse a la nación que constituye la unidad más amplia, como ex gente Asturum o ex gente Cantabrorum, el término puede haber conocido variantes de utilización para referirse a unidades étnicas de menos envergadura, que la escasez de datos nos impide detectar con claridad. Por ejemplo, solamente se emplea una vez entre los cántabros orgenomescos, en un epígrafe de Parres (Oviedo) que dice 
Bovecio Bode Cives Org(e)nomesc(-) Ex Gent(e) Pembelor(vm) (CIL II, 5729). ${ }^{24}$

La forma PEMBELOR(VM) plantea más problemas de los que parece, puesto que, con independencia de que se acepte o no la existencia de una unidad familiar BoDECIVES(CVM), resulta muy dudoso que se pueda interpretar *pembelo- como derivado de un antropónimo. Ya hace mucho tiempo, A. Tovar (1954) propuso una interpretación prima facie, que consistía en ver aquí un derivado ordinal del numeral indoeuropeo *penk ${ }^{w} e$ "cinco", con fonética de "tipo britónico". Hay que entender que hace medio siglo prácticamente nada se sabía sobre las lenguas celtas de la Península Ibérica. Lo que ya no resulta tan comprensible es que esta interpretación siga gozando de cierta aceptación hasta el día de hoy. Efectivamente, *pempe podría ser la forma que exhibe el numeral "cinco" en un dialecto celta peninsular con labialización de labiovelares. Además, un derivado suyo podría emplearse como etnónimo (como sucede con los Vocontī galos), en el entendido de que se refiere al número de batallones o de subdivisiones étnicas con que cuenta ese grupo en particular. Sin embargo, y siempre en el entendido de que la lectura es correcta, cuesta entender el empleo de un sufijo endocéntrico de valor diminutivo, que vendría a significar "los cinquillos", ya que no es fácil encontrar -lo- documentado como elemento de derivación a partir de numerales. ${ }^{25}$ Otra cosa muy diferente sería que se modificara la lectura a favor de PEMBEIORVM, lo que permitiría reconstruir una protoforma *pempe-yo- comparable al itálico Pompeius.

Existe una alternativa inexplorada que me parece algo más plausible: Que se trate de un compuesto *penno-belo-, de * $k^{w} e n n o-$ "cabeza" y * gwelHo- "poderoso, grande", que significa "de cabeza grande" (o, literalmente, "grande de cabeza"). En las lenguas celtas existen varios compuestos cuyo primer elemento es $* k^{w} e n n o-$, como galo Пevvoouıvoos "de cabeza blanca" y sus correspondencias. ACS II, col. 964,

${ }^{24} \mathrm{Si}$ se acepta esta división de palabras, y no la que se propuso en su momento por el editor (cf. ERA, nr. 36), y que consistía en interpretar toda la secuencia BoDECIVES como abreviatura de un nombre de familia BoDECIVES(CVM). No es imposible, dado que se documenta un BodivesC(VM) en un epígrafe de Sorribas (León).

25 Osco pumperias, umbro pumperias, lat. Pompilius proceden de un sufijo complejo secundario -el-yo-o, más probablemente, del productivo-ed-yo-, con alteración en la forma latina. 
cita un lugar llamado Pembeciacum, ${ }^{26}$ que hace derivar de *pennobekko- "pequeño de cabeza" y que estaría en la base de Pembé (Loire Inférieure). Está bien establecido que a la hora de reconstruir el árbol genealógico celta, la evolución $/ \mathrm{k}^{\mathrm{w}} />/ \mathrm{p} /$ es prácticamente inutilizable. Que al menos en posición inicial se diera semejante evolución en el dialecto de los orgenomescos carece por sí mismo de relevancia para establecer su posición dialectal.

\section{BIBLIOGRAFÍA}

Albertos Firmat, Ma L. (1985), "La onomástica personal indígena del Noroeste peninsular (astures y galaicos)”, J. De Hoz, ed., Actas del III Coloquio sobre Lenguas y Culturas Paleohispánicas, Salamanca, pp. 255-310.

Alföldi, A. (1975), Reseña de St. Weinstock, Divus Iulius (1971), Gnomon 47, pp. 154-79.

Allen, W. Sidney (reimpr. 1991), Vox Graeca. The pronunciation of Classical Greek, Cambridge, Cambridge University Press.

ASOCIACIÓN ACADÉMICA INTERNACIONAL (1991), Tabula Imperii Romani, Hoja K-29: Porto, Madrid: C.S.I.C. [TIR-K].

Beltrán Lloris, F. (1992), "Culto a los lares y grupos de parentesco en la Hispania indoeuropea”, M. Mayer - J. Gómez Pallares, eds., Religio Deorum. Actas del Coloquio Internacional de Epigrafía «Culto y Sociedad en Occidente», Barcelona, pp. 59-71.

Benveniste, É. (1965), "Termes de parenté dans les langues indoeuropéennes", L'Homme 5, pp. 5-16.

Bragado Toranzo, J. M. - García Martínez, S. M. (1997), "Una nueva deidad indígena del Conventus Asturum”, Studia Zamorensia 4, pp. 21-30.

Búa CARballo, J. C. (1997), "Dialectos indoeuropeos na franxa occidental hispánica", G. Pereira, ed., Galicia Fai Dous Mil Anos. O Feito Diferencial Galego I, Santiago, pp. 51-99.

Canto, A. M. - Bejarano, A. - Palma, F. (1997), "El mausoleo del Dintel de los Ríos de Mérida, Revve Anabaraecus y el culto a la confluencia", Madrider Mitteilungen 38, pp. 247-94.

Delamarre, X. (2002²), Dictionnaire de la langue gauloise, París, Errance.

Devoto, G. (1967), "Leponzi”, Scritti minori, pp. 324-35.

Diego Santos, F. (1985), Epigrafía romana de Asturias, Oviedo [ERA].

ENCARNAÇÃo, J. D' - (1975), Divindades indigenas sob o dominio romano em Portugal, Lisboa, Imprensa Nacional. [DIP].

26 Sin fuente; probablemente no es, por lo tanto, más que una reconstrucción culta extraída de algún repertorio. 
ESKA, J. F. (1995), "Resyllabification and epenthesis in Hispano-Celtic", Journal of Celtic linguistics 5, pp. 71-89.

García Alonso, J. L. (2003), La Península Ibérica en la Geografía de Claudio Ptolomeo, Anejos de Veleia, Vitoria, Universidad del País Vasco.

- (2007), "Ethnic names in Hispania", J. L. García Alonso, ed., Celtic and its neighbouring languages, Salamanca, pp. 83-100.

García Rozas R. - ABÁsolo J.-A. (en prensa), “Algunas aportaciones al conocimiento del panteón indígena en el Occidente peninsular”, Congreso sobre Religiones, 1995, Sintra.

González Rodríguez, Ma C. (1997), Los astures y los cántabros vadinienses, Anejos de Veleia 10, Vitoria, Universidad del País Vasco.

GonZÁlez RodríGuez, Ma C. - SANTOS, J. (1987), "El caso de las llamadas gentilitates:

Revisión y propuestas", J. Gorrochategui - J. L. Melena - J. Santos, eds., Studia

Palaeohispanica. Actas del IV Coloquio sobre Lenguas y Culturas Paleohispánicas, Vitoria, pp. 373-82.

Holder, A. (1896-1922), Alt-Celtischer Sprachschatz I-III, Leipzig. [ACS].

LAmbert, P.-Y. (1980), "Étymologies: 4. Irlandais súil”, Études Celtiques, 17, 175-78.

Liddell, H. G. - ScotT, R. - JonEs, H. S. (1968), A Greek-English Lexicon, Oxford, Clarendon Press.

MAÑCZAK, W. (2006), "Lusitanien REVE = Latin JOVI”, Lingua Posnaniensis 48, pp. 63-65.

Melchert, H. C. (1994), Anatolian historical phonology, Amsterdam-Atlanta, Rodopi. POKORNY, J. (1959), Indogermanisches Etymologisches Wörterbuch, Berna, Francke. $[I E W]$.

PRósPer, B. Ma (2002), Lenguas y religiones prerromanas del Occidente de la Península Ibérica, Salamanca, Universidad de Salamanca.

- (2004), "Varia Palaeohispanica Occidentalia I. Lusitano TAVRom IfADEM. II Origen del topónimo galaico $\Gamma \lambda \alpha \nu \delta o ́ \mu \iota \rho o v$. III. Indoeuropeo *kor-(y)o- «ejército» en Hispania. IV. Un superlativo hispano-celta *Kintúsamos y una cuestión de acento", Palaeohispanica 4, pp. 169-94.

RAMÍREZ SÁNCHEZ, M. (2001), Epigrafía y organización social en la región celtibérica: Los grupos de parentesco, Las palmas de Gran Canaria, ed. en microfichas.

REDEntor, A. (2002), Epigrafia romana na região de Bragança, Lisboa, Instituto Português de Arqueologia.

- (2006), "Manifestações religiosas e onomástica na Civitas Zoelarum", Conimbriga 45 , pp. 253-73.

Richard, J.-C. (1986), "Sur une triple étiologie du nom Iulus: Seru., Aen. 1, 267 et Ps. Aur. Vict., Orig. 15, 5”, Festschrift für G. Radke, Münster, pp. 232-244.

- (1983), "Sur une triple étiologie du nom Iulus, II”, REL, 61, pp. 108-121.

RodríGueZ, P. (1994), "Los términos gens y gentilitas en los escritores latinos", $\mathrm{M}^{\mathrm{a}} \mathrm{C}$.

González - J. Santos, eds., Las estructuras sociales indígenas del Norte de la

Península Ibérica, Vitoria, pp. 67-72.

Solinas, P. (1995), "Il celtico in Italia", Studi Etruschi 60, pp. 311-408.

Solmsen, F. (1894), Studien zur lateinischen Lautgeschichte, Estrasburgo, Trübner. 
SZEMERÉNYI, O. (1989), An den Quellen des lateinischen Wortschatzes, Innsbruck, IBS. Tovar, A. (1954), "Numerales indoeuropeos en Hispania”, Zephyrus 5, pp. 17-22.

Untermann, J. (2000), Wörterbuch des Oskisch-Umbrischen, Heidelberg, Winter.

VALlEJo RuIZ, J. Ma (2005), La antroponimia indígena de la Lusitania romana, Anejos de Veleia, Vitoria, Universidad del país Vasco.

VILlar, F. (1996), "El teónimo lusitano Reve y sus epítetos", W. Meid - P. Anreiter, eds., Die Grösseren Altkeltischen Sprachdenkmäler, Innsbruck, pp. 160-211.

Villar, F. - Díaz Sanz, Ma A. - Medrano Marqués, M. Ma - Jordán Cólera, C. (2000), El IV Bronce de Botorrita (Contrebia Belaisca), Salamanca, Universidad de Salamanca. [BB.IV].

VILlar, F. - Prósper, B. Ma (2005), Vascos, celtas e indoeuropeos. Genes y lenguas, Salamanca, Universidad de Salamanca.

VINE, B. (1993), Studies in Archaic Latin inscriptions, Innsbruck, IBS.

Walde, A. - Hofmann, J. B. (1965), Lateinisches Etymologisches Wörterbuch, Heidelberg, Winter. [LEW].

Weinstock, ST. (1971), Divus Julius, Oxford, Clarendon Press.

WITCZAK, K. T. (1999), "On the Indo-European origin of two Lusitanian theonyms (LAEBO and ReVE)", Emerita 67, pp. 65-73.

- (2005), Jezyk i religia luzytanów, Lódź, Wydawnictwo Uniwersytetu Lódźkiego. 